\title{
Negative Ion Motion in Normal and Superfluid ${ }^{3} \mathrm{He}^{*}$
}

\author{
A. I. Ahonen, J. Kokko, M. A. Paalanen, \\ R. C. Richardson, $\nmid$ W. Schoepe,,$\div$ and Y. Takano \\ Low Temperature Laboratory, Helsinki University of Technology, Espoo, Finland
}

(Received July 6, 1977)

We report the first measurements of negative ion motion in the superfluid phases of ${ }^{3} \mathrm{He}$ and in the normal phase below $17 \mathrm{mK}$. Refrigeration was achieved with nuclear demagnetization of copper and we used a pulsed NMR platinum powder thermometer immersed in the liquid. In the A phase the longitudinal resonance frequency provided an additional high-resolution thermometer. In the normal phase we observed a strictly temperatureindependent mobility. In the superfluid phases we found two velocity regimes. For small applied electric fields the velocity is a linear function of the field and the corresponding mobility increases monotonically toward lower temperatures. At high electric fields the velocity is a nonlinear function of the field as a result of the pair-breaking effect of the moving ion. Available theoretical calculations are only in partial agreement with our results.

\section{INTRODUCTION}

Ions have proven a very useful tool in studying the properties of superfluid ${ }^{4} \mathrm{He}$. They are atomic size probes, which are readily detectable even in small concentrations because of their charge. They are also easy to act on with an external electric field. In this paper we present the first negative ion experiments carried out in superfluid ${ }^{3} \mathrm{He}$ and in normal ${ }^{3} \mathrm{He}$ below $17 \mathrm{mK}$. Some of the experimental data have already been reported in a brief form. ${ }^{1}$

The negative ion in liquid helium is a self-trapped electron localized inside an otherwise empty bubble which represents a spherical square-well potential for the electron. The radius of the bubble varies with the pressure around $15 \AA$ and the strength of the potential is roughly $1 \mathrm{eV} \cdot{ }^{2,3,} \S$ The

* This work was supported financially by the Academy of Finland.

† Guggenheim Fellow on leave from Cornell University, Ithaca, New York.

\$ On leave from Regensburg University, Regensburg, West Gcrmany, supported by Deutsche

Forschungsgemeinschaft and Finnish Ministry of Education.

$\S$ A summary of recent measurements is found in Ref. 4 . 
diameter of the negative ion is smaller than the mean free path of the excitations in liquid ${ }^{3} \mathrm{He}$ at millikelvin temperatures and also shorter than the coherence length of the superfluid state of ${ }^{3} \mathrm{He}$.

An ion moving through liquid helium experiences a drag force due to the interaction with the excitations in the liquid. At relatively high temperatures $(T \simeq 2 \mathrm{~K})$ the mean free path in the bulk fluid is shorter than the ionic radius, so that Stokes' law for viscous drag on a sphere provides an adequate description of the motion. ${ }^{2}$ As the temperature falls, however, the increasing mean free path requires a more fundamental approach and the drag must be considered as arising from collisions with individual quasiparticles. A measurement of the drag force thus provides direct information about the excitation spectrum of the fluid.

In the present work we have measured the drift velocity $v$ of the ion in an applied electric field $\mathscr{E}$. For small electric fields the velocity is directly proportional to $\mathscr{E}$ and the mobility is defined as $\mu=v / \mathscr{E}$. At high electric fields the velocity deviates from linear behavior. Also, this regime is worth investigating since additional information about the excitation spectrum can be obtained.

In the case of ${ }^{4} \mathrm{He}$, the superfluid state has been investigated in both the low- and the high-velocity regimes. The mobility data above $0.8 \mathrm{~K}$ can be adequately understood in terms of roton scattering. ${ }^{5}$ At lower temperatures, between 0.4 and $0.5 \mathrm{~K}$, a mobility limited by phonon scattering is found. ${ }^{5,6}$ The hydrodynamic structure of the superfluid has been studied in the high-velocity regime. Rayfield and $\operatorname{Reif}^{7}$ were the first to create and detect quantized vortex rings by ions. Negative ions have also been used to produce rotons 8 above 12 bar, making possible studies of the Landau critical velocity, ${ }^{9}$ which has not been seen with hydrodynamic flow measurements.

Prior to our work, the mobility of the negative ion ${ }^{10-12}$ in ${ }^{3} \mathrm{He}$ had been measured down to $17 \mathrm{mK}$. We have extended the range in the normal fluid down to the superfluid transition temperature $T_{c}$ and have studied the ion behavior in the superfluid phases of ${ }^{3} \mathrm{He}$ at several pressures in both the linear and the nonlinear regimes of the drift velocity.

In the next section we discuss our apparatus and the experimental procedure. In Section 3 we present our results in the normal phase below $35 \mathrm{mK}$ and discuss the current theoretical understanding of the ion motion. Finally, the superfluid results are presented and compared with recent theoretical calculations in Section 4.

\section{EXPERIMENTAL TECHINIQUES}

Our cooling method was nuclear demagnetization of copper, which has been discussed in detail elsewhere. ${ }^{13}$ Nuclear cooling is a single-cycle 
method, which in our case gave about $20 \mathrm{~h}$ below the superfluid transition temperature, the nuclear stage warming up because of an external heat leak of $5 \mathrm{nW}$. The drifting temperature forced us to use a fast measuring technique of the ion velocity. Another limitation was the heating by the ion production, which can shorten the warmup time if it becomes comparable to the residual heat leak. It also raises the temperature of the ${ }^{3} \mathrm{He}$ liquid above the temperature of the chamber walls as a result of the large Kapitza resistance between them. This can prevent ${ }^{3} \mathrm{He}$ from reaching temperatures below $1 \mathrm{mK}$.

There exist at least three different methods of producing ions in liquid helium: ionization by a radioactive source, ${ }^{*}$ field emission and field ionization by a high electric field at a sharp metal tip, ${ }^{15,16}$ and injection of electrons by thin film electron emitters. ${ }^{17,18}$ (The last method can produce only negative ions.) We can compare the suitability of these methods for millikelvin experiments by estimating the heat input per $1 \mathrm{pA}$ ion current. A tritiated titanium source of a few $\mathrm{mCi}$, which has been used in ${ }^{3} \mathrm{He}$ down to $17 \mathrm{mK},{ }^{10,11}$ gives a $10^{-15}-10^{-13}$ A current and a heat input of $100 \mathrm{nW} .^{10}$ This represents a heat leak of about $1 \mu \mathrm{W} / \mathrm{pA}$. The thin-film electron emitter works with an $8 \mathrm{~V}$ bias and with an efficiency, i.e., cmitted current compared with total conduction current, of at best only $5 \times 10^{-4}{ }^{18}$ This gives a heat input of $16 \mathrm{nW} / \mathrm{pA}$. Field emission and field ionization need a voltage threshold of about $500 \mathrm{~V}$ for any current production at all, implying $0.5 \mathrm{nW} / \mathrm{pA}$.

A radioactive source cannot be shut off and it generates the largest heat leak of the three. The other two methods can be used in a pulsed mode, which considerably reduces the heating. We used the field emission/ionization technique in the pulsed mode.

The experimental ${ }^{3} \mathrm{He}$ chamber is shown in Fig. 1. The sintered $\mathrm{Cu}$ sponge provided a $10-15 \mathrm{~m}^{2}$ contact area $\dagger$ between ${ }^{3} \mathrm{He}$ and the copper nuclear cooling stage, which is not shown in the figure. All the measuring probes were attached to the lid of the chamber, which was exchangeable. The ion velocity spectrometer was in the free space below the lid. The NMR coils for temperature measurements were located on the axis of the sample chamber in a hole drilled into the sintered copper sponge.

We determined the temperature of the ${ }^{3} \mathrm{He}$ by measuring the nuclear magnetization of finely divided platinum powder immersed in the liquid, using a commercial NMR spectrometer $\$$ operating at a static field of $28 \mathrm{mT}$.

\footnotetext{
*A summary of different methods of producing ions in liquid helium is given by Moss. ${ }^{14}$ tThe previously reported value $\mathrm{e}^{13}$ for this area was $30 \mathrm{~m}^{2}$ based on measurements of nitrogen adsorption on a replica specimen. The present value is based upon a direct $B E T^{19}$ measurement.

tInstruments for Technology, Espoo, Finland.
} 


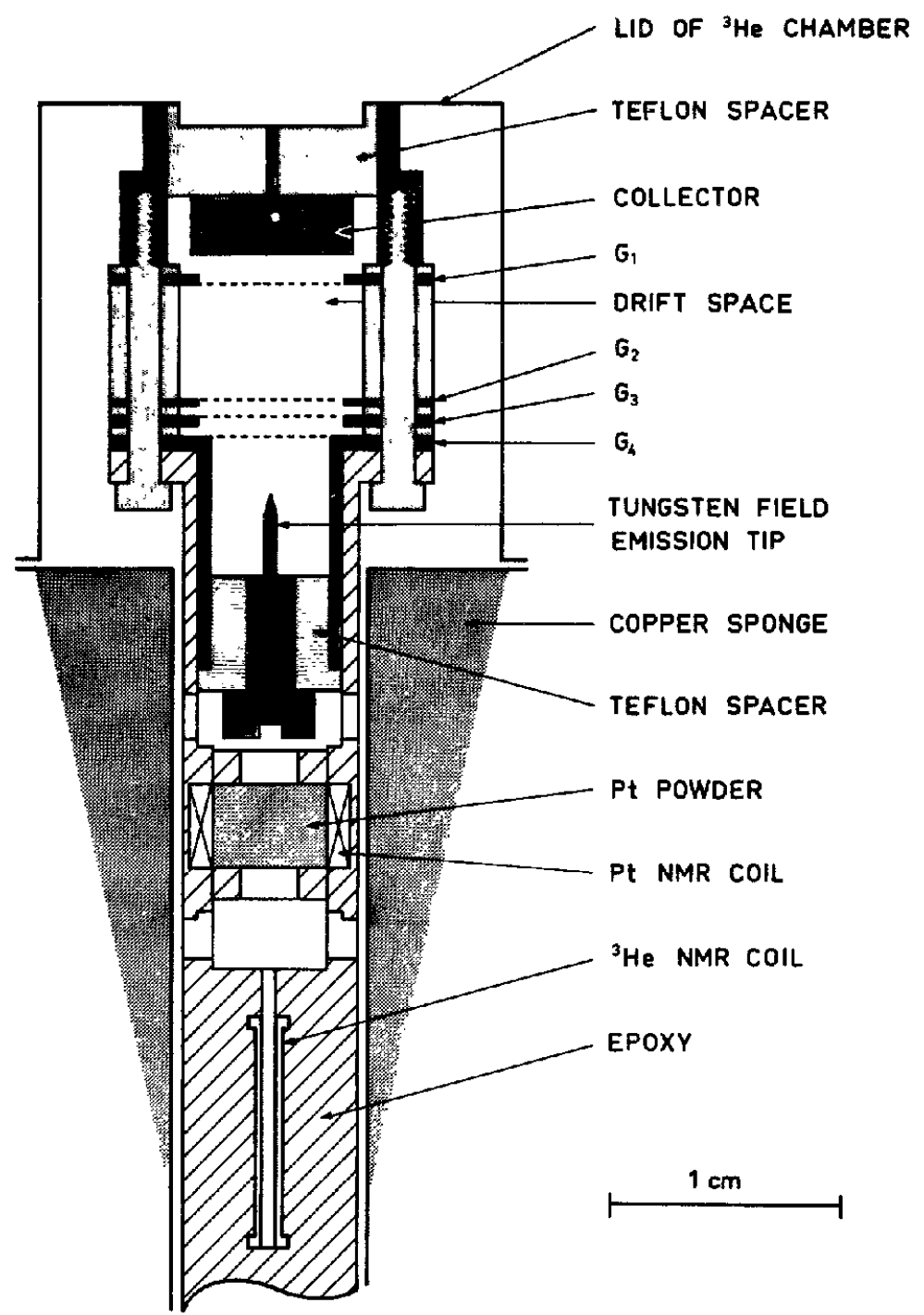

Fig. 1. Schematic illustration of the velocity spectrometer and the thermometers in the ${ }^{3} \mathrm{He}$ chamber.

For a detailed discussion we refer to earlier publications. ${ }^{20,21}$ The resolution of the Pt thermometer was about $0.5 \%$.

An improvement in the temperature resolution can be achieved in the A phase by using the shift of the transverse resonance frequency as a secondary thermometer. For small tipping angles the transverse resonance 
frequency $\nu$ obeys the relation ${ }^{22}$

$$
\nu^{2}=\left(\gamma H_{0} / 2 \pi\right)^{2}+\nu_{L}^{2}\left(P, T / T_{c}\right)
$$

where $\gamma H_{0} / 2 \pi$ is the frequency in the normal liquid and $\nu_{L}\left(P, T / T_{c}\right)$ is the characteristic longitudinal frequency of the $A$ phase, which depends on pressure and temperature. The ${ }^{3} \mathrm{He}$ signal came from the liquid in a slab of rectangular cross section of $0.6 \mathrm{~mm} \times 5.0 \mathrm{~mm}$, located below the Pt thermometer. At each pressure, $\nu_{L}\left(P, T / T_{c}\right)$ was calibrated against the Pt thermometer and the calibration agreed well with our earlier $\mathrm{cw}$ NMR determinations. ${ }^{23}$ Changes in the transverse resonance frequency could be resolved to within $10 \mathrm{~Hz}$, corresponding to a temperature resolution of $0.05 \%$ in a magnetic field of $28 \mathrm{mT}$. As the "shift thermometer" felt the temperature of the ${ }^{3} \mathrm{He}$ directly, it had the additional advantage of a practically instantaneous response.

In the A phase the frequency shift is a property of the bulk liquid. In the $B$ phase the situation is more complicated because there is no bulk frequency shift. In restricted geometries the walls and a magnetic field together can orientate the liquid textures, causing a frequency shift. Due to the bending of the textures, it was impossible to take advantage of shift thermometry in the present geometry, however, and we used only the Pt thermometer in the B phase.

The tungsten tip for the field emission and field ionization was made of $60 \mu \mathrm{m}$ wire electrochemically etched in $1 \mathrm{M} \mathrm{NaOH}$ solution. A typical radius of such a tip is $700 \AA$ as measured with an electron microscope. The current-voltage characteristic had a reasonably stable threshold at about $300 \mathrm{~V}$ and the tip was operated just above the threshold, giving a $1 \mathrm{pA}$ output current. In the pulsed mode the current was cut off by reducing the tip voltage $10 \%$ below the threshold. The tip was on for $100 \mathrm{msec}$ at a time with a repetition rate between 0.5 and $1 \mathrm{~Hz}$.

The heating per one $100 \mathrm{msec}$ pulse was less than $0.1 \mathrm{~nJ}$, which we could not detect in the warmup rate. However, at $T_{c}$, temperature differences typically as large as $2 \mu \mathrm{K}$ were observed between the velocity spectrometer and the "shift thermometer." The stability of the tip current in the pulsed mode was good over a period of several hours. Operating the tip as little as $15 \mathrm{~V}$ above the threshold produced significant heating because of the increased current.

We used the "single-pulse" time-of-flight method to measure the ion velocity. An ion cloud was produced by the high-voltage pulse between the tungsten tip and grid G4 (cf. Fig. 1). G3 was used as a gate by raising or lowering its potential with respect to the potential of G2. The velocity of the ion cloud was obtained by measuring the time of flight of the ions over the 5 - mm drift space between G2 and G1. The ion pulse was detected by the 


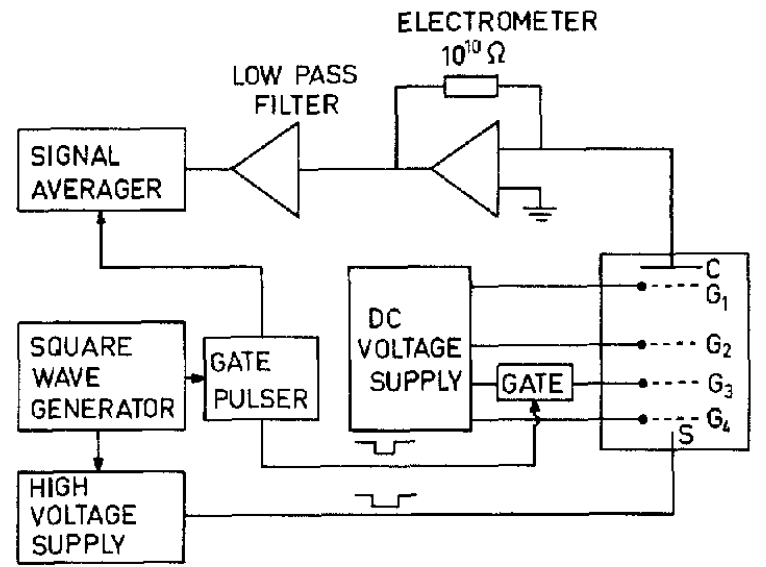

Fig. 2. Block diagram of the velocity spectrometer. Typical values of the voltages used were $V_{C}=0, V_{G 1}=-25 \mathrm{~V}$, $V_{G 2}=-35 \mathrm{~V}, V_{G 3}$ (open) $=-55 \mathrm{~V}, V_{G 3}$ (closed) $=-25 \mathrm{~V}$, $V_{\mathrm{G} 4}=-75 \mathrm{~V}$, and $V_{\mathrm{S}}=-400 \mathrm{~V}$.

collector $\mathrm{C}$. The grids were made out of 30 lines $/ \mathrm{cm}$ Cu mesh. The chamber walls and the electrodes were gold-plated to reduce static charge buildup.

The electronics for the ion velocity measurements are shown in Fig. 2. A square wave generator was used as a timer for the high-voltage supply and the gate pulser. The output of the gate pulser driving the potential of grid G3 had a manually adjustable delay and pulse length. The ion pulse arriving at collector $\mathrm{C}$ was detected by a fast electrometer consisting of an $\mathrm{AD} 41 \mathrm{~L}$ operational amplifier and a $10^{10}-\Omega$ feedback resistor. A Nicolet 1074 signal averager* collected approximately 10-20 current pulses for one velocity measurement.

Figure 3 shows the relevant times of the velocity measurement. A high-voltage pulse from time $t_{1}$ to time $t_{4}$ produced an ion cloud whose edges were rather rounded. The ion cloud was then shaped with the aid of G3, cutting a sharp front edge at time $t_{2}$ and also a sharp trailing edge at $t_{3}$. This was brought about by holding G3 at a lower potential than G2, stopping the ions at G3. At time $t_{2}$ the potential was raised above the potential of $\mathrm{G} 2$ and the sharp front edge started from G3. At $t_{3}$ the potential was changed back to the initial value and the trailing edge left $\mathrm{G} 2$.

The lowest part of Fig. 3 shows the signal as seen on the display of the signal averager. The spikes at times $t_{1}, t_{2}, t_{3}$, and $t_{4}$ come from the high-voltage and gate pulses. In the trapezoidal signal we could distinguish

*Nicolet Instrument Corp., Madison, Wisconsin. 

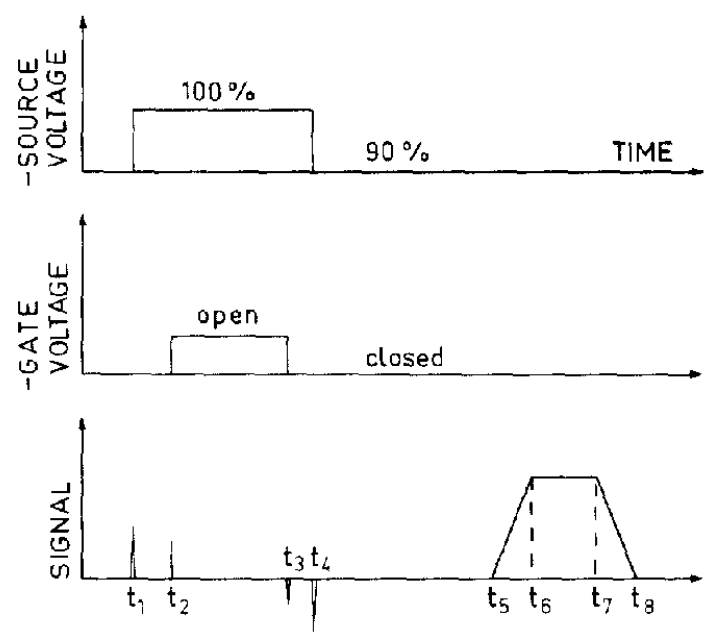

Fig. 3. Timing of the source and the gate voltages. In the electrometer signal we can also see the spikes before the trapezoidal ion pulse.

four relevant times. At $t_{5}$ the front edge arrived at grid 1 , at $t_{6}$ it arrived at the collector, and the same happened to the trailing edge at times $t_{7}$ and $t_{8}{ }^{*}$

The trailing edge traveled across the drift space from $\mathrm{G} 2$ to $\mathrm{G} 1$ in the time interval $t_{7}-t_{3}$. Under ordinary circumstances that time was sufficient for measuring the drift velocity. However, for small electric fields the ion cloud was considerably expanded due to the space charge effect, making the trailing edge travel more slowly than the front edge. To correct for this space charge effect we also measured the time $t_{5}-t_{2}$, which was the time of travel of the front edge from G3 to G1. The time spent between G3 and G2 was measured separately by determining the minimum gate length $t_{3}-t_{2}$ that allowed the ions to reach $\mathrm{G} 2$ and this time was subtracted from $t_{5}-t_{2}$. The corrected time of flight inferred from both the front edge and the trailing edge is calculated in the appendix.

With this apparatus we could detect ion signals only for electric fields above $10 \mathrm{~V} / \mathrm{cm}$ in the normal fluid and above $3 \mathrm{~V} / \mathrm{cm}$ at the lowest temperatures in the $\mathrm{B}$ phase. This was probably related to the abovementioned space charge effect which decreased the signal amplitude. Also, electric field inhomogeneities in the drift space and ion leakage to the walls and grids may have made the signal undetectable for small electric fields.

\footnotetext{
${ }^{*}$ For very short gate pulses it may happen that the trailing edge arrives at G1 before the front edge reaches $\mathrm{C}$ and the meaning of the times $t_{6}$ and $t_{7}$ in Fig. 3 is reversed. Care was taken to distinguish these two modes.
} 
Another limitation of the present apparatus was the horizontal direction of the static magnetic field for NMR measurements. In the A phase the magnetic field thus oriented the angular momentum $l$ of the Cooper pairs parallel to a vertical plane. Since the ions were moving in the vertical direction, the angle between $\boldsymbol{l}$ and the drift velocity $\mathbf{v}$ was not well defined; therefore we could not determine a possible anisotropy of the mobility. Only a magnetic field along the ion path could have given the uniquely defined perpendicular component of the mobility tensor in the A phase.

\section{MOBILITY OF NEGATIVE IONS IN NORMAL ${ }^{3} \mathrm{He}$}

From earlier experiments the mobility of negative ions in ${ }^{3} \mathrm{He}$ was reasonably well known down to $17 \mathrm{mK}^{10-12}$ and the different measurements were in agreement with each other. Below $100 \mathrm{mK}$ the mobility had been found to be temperature independent. The pressure dependence was understood: Toward higher pressures the mobility increased, an effect which can be explained by the shrinking bubble.

We have measured the mobility down to $T_{c}$ and observed that it remains temperature independent even below $17 \mathrm{mK}$ at all pressures. Our result at 28.4 bar is shown in Fig. 4 . The measurement was carried out in a constant electric field of $60 \mathrm{~V} / \mathrm{cm}$.

In order to understand the temperature independence, we review the current theory of mobility of an impurity in a Fermi liquid. Let us first assume that the ions behave like free particles. After colliding with a ${ }^{3} \mathrm{He}$ quasiparticle, the ion gets an additional momentum $\hbar \mathbf{K}$ and the mean recoil energy is ${ }^{2}$

$$
\left\langle E_{\text {rec }}\right\rangle=\hbar^{2} K^{2} / 2 M^{*}
$$

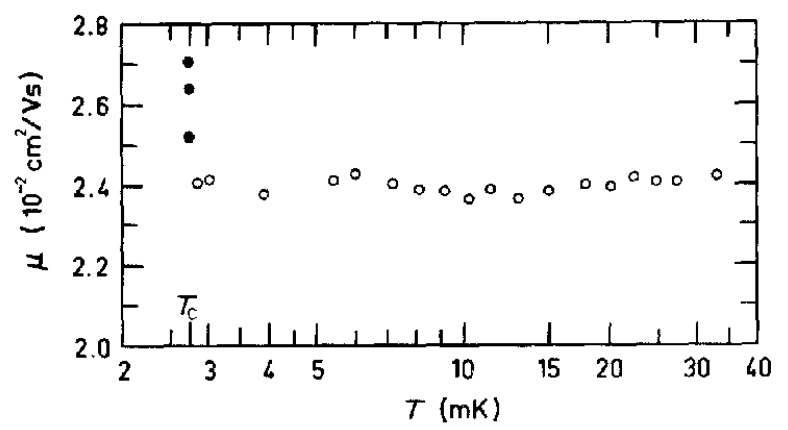

Fig. 4. Mobility of the negative ion in normal ${ }^{3} \mathrm{He}$ at 28.4 bar as a function of temperature: $(O)$, normal phase; $(0)$, A phase. There is obviously no change in the mobility until $T_{c}$. 
where $M^{*}$ is the effective mass of the ion. In the Fermi liquid $K$ is of the order of Fermi momentum $k_{\mathrm{F}}$ and the recoil energy is equal to the thermal energy $k_{\mathrm{B}} T$ at a characteristic temperature

$$
T_{0}=\hbar^{2} k_{\mathrm{F}}^{2} / 2 M^{*} k_{\mathrm{B}}=\left(m^{*} / M^{*}\right) T_{\mathrm{F}}
$$

where $m^{*}$ is the effective mass of a ${ }^{3} \mathrm{He}$ quasiparticle and $T_{\mathrm{F}}$ is the Fermi temperature. Far above $T_{0}$ the recoil energy is small compared with the thermal energy, and we may neglect it and use an elastic scattering model, where $M^{*}$ is considered infinite. The problem is then identical to impurity scattering in metals where electrons are deflected by defects bound to a solid lattice, the lattice providing the infinite mass. The resulting expression for the mobility, well below $T_{\mathrm{F}}$, is temperature independent

$$
\mu=e / \sigma n p_{\mathrm{F}}, \quad T \gg T_{0}
$$

where $e$ is the electronic charge, $n$ is the number density of ${ }^{3} \mathrm{He}$ atoms, and $\sigma$ is the scattering cross section.

Below $T_{0}$ the recoil energy exceeds the thermal energy and the resulting mobility ${ }^{24}$ should be proportional to $T^{-2}$,

$$
\mu=\frac{8}{\pi^{2}} \frac{e}{\text { onp }_{\mathrm{F}}}\left(\frac{T_{0}}{T}\right)^{2}, \quad T \ll T_{0}
$$

$T_{0}$ is about $100 \mathrm{mK}$ at 28.8 bar and $17 \mathrm{mK}$ at 0 bar (Table II). The earlier measurements ${ }^{10}$ were carried out down to $T / T_{0} \sim 0.3$ only. In our measurement the lowest $T / T_{0}$ was 0.03 and the constant mobility clearly reveals the failure of formula (5) in the case of negative ions. $\dagger$

In a more fundamental treatment, Josephson and Lekner ${ }^{2.5}$ described the mobility in terms of temporal correlations of the ion's movements. They obtained

$$
\frac{1}{\mu}=\frac{\pi^{2} n p_{\mathrm{F}}}{2 e} \int_{0}^{\pi} d \theta(\sin \theta)(1-\cos \theta) \sigma(\theta) \int_{-\infty}^{\infty} d \tau \frac{F_{0}(K, \hbar \beta \tau)}{\cosh ^{2}(\pi \tau / 2)}
$$

Here $\sigma(\theta)$ is the differential scattering cross section, $\theta$ is the scattering angle, $K=2 k_{\mathrm{F}} \sin \left(\frac{1}{2} \theta\right), \tau=t / \hbar \beta, \beta=1 / k_{\mathrm{B}} T$, and $F_{0}(K, t)$ contains the information about the motion of the ion over a time interval $t$. Here $F_{0}(K, t)$ is an ensemble average

$$
F_{0}(K, t)=\langle\exp [-i \mathbf{K} \cdot \mathbf{r}(0)] \exp [i \mathbf{K} \cdot \mathbf{r}(t)]>
$$

with $\mathbf{r}(t)$ the Heisenberg operator for the ion's position.

In the case of positive ions the mobility increases below $T_{0}$ toward lower temperatures; see Ref. 10. 
The zero-time value of $F_{0}$ is 1 . If $F_{0}(K, t)$ is decaying fast at $|t|<\hbar \beta$, the mobility will increase; this can be seen from the $\tau$ integral in Eq. (6). $F_{0}(K, t)$ decays more slowly for a diffusing ion than for a free ion (Table I) and one is forced to assume diffusive motion of the ion in order to explain why the mobility is temperature independent. In fact the ion will move ballistically starting at some time $t=0$ for a period of time

$$
\tau^{*}=M^{*} \mu / e
$$

but later the motion is diffusive. If $\hbar \beta \geqslant \tau^{*}$, one has to use $F_{0}(K, t)$ of the diffusing ion in Eq. (6). For negative ions in ${ }^{3} \mathrm{He}$ this implies any temperature below $0.3 \mathrm{~K}$.

In Table I we have listed $F_{0}$ in the cases of a free ion, an elastically scattering ion $\left(M^{*}=\infty\right)$, and a diffusing ion in the Gaussian approximation. ${ }^{26} D$ is the diffusion constant, related to $\mu$ by the Einstein relation $D=\mu k_{\mathrm{B}} T / e$. The scattering function $S_{0}(K, \omega)$ is the Fourier transform of $F_{0}(K, t)$ and gives the spectrum of energy absorbed by the ion when it is given the momentum $\hbar \mathbf{K}$. The width of the absorption peak $\Delta E_{\text {rec }}$ is a measure of the elasticity of this collision. For diffusitive motion the width is proportional to temperature and for negative ions at low temperature the width is much smaller than the thermal energy (Table II)

$$
\Delta E_{\mathrm{rec}} \sim\left(\hbar K_{\mathrm{F}}^{2} \mu / e\right) k_{\mathrm{B}} T
$$

Because of the narrow width the scattering is almost elastic in the diffusion model.

Fetter and Kurkijärvi, ${ }^{26}$ who generalized the Josephson-Lekner formalism to finite velocities, assumed $F_{0}(K, t)=\exp \left(-K^{2} D|t|\right)$ as a first

\begin{tabular}{|c|c|c|c|}
\hline & $F_{0}(K, t)$ & $S_{0}(K, \omega)$ & $\Delta E_{\text {rec }}$ \\
\hline Free ion & $\exp \left(-\frac{k_{\mathrm{B}} T K^{2}}{2 M^{*}} t^{2}-i \frac{\hbar K^{2}}{2 M^{*}} t\right)$ & $\left(\frac{M^{*}}{2 \pi k_{\mathrm{B}} T K^{2}}\right)^{1 / 2}$ & $\sim\left(\frac{k_{\mathrm{B}} T \hbar^{2} K^{2}}{M^{*}}\right)^{1 / 2}$ \\
\hline $\begin{array}{l}\text { Elastic } \\
\text { scattering }\end{array}$ & 1 & $\delta(\omega)$ & 0 \\
\hline $\begin{array}{l}\text { Diffusive } \\
\text { motion }\end{array}$ & $\exp \left(-K^{2} D|t|\right)$ & $\frac{K^{2} D / \pi}{\omega^{2}+\left(K^{2} D\right)^{2}}$ & $\sim \hbar K^{2} D$ \\
\hline
\end{tabular}

TABLE I 
TABLE II

\begin{tabular}{ccccl}
\hline$P$, bar & $R, \AA$ & $M^{*},{ }^{a} m_{3-\mathrm{He}}$ & $T_{0, \mathrm{mK}}$ & $\hbar k_{\mathrm{F}}^{2} \mu / e$ \\
\hline 0 & 20.3 & 290 & 17 & 0.038 \\
28.8 & 10.8 & 61 & 100 & 0.12 \\
\hline${ }^{a} M^{*}=\frac{1}{2}\left(4 \pi R^{3} / 3\right) n m_{3-\mathrm{Hc}}{ }^{2}$
\end{tabular}

correction to the elastic scattering model and obtained

$$
e \mathscr{E}=\sigma_{1} n p_{\mathrm{F}}\left\{\left(1-0.883 \frac{\hbar k_{\mathrm{F}}^{2} \mu}{e} \frac{\sigma_{2}}{\sigma_{1}}\right) v+\left[0.0185 \frac{\hbar k_{\mathrm{F}}^{2} \mu}{e}\left(\frac{p_{\mathrm{F}}}{k T}\right)^{2} \frac{\sigma_{3}}{\sigma_{1}}\right] v^{3}\right\}
$$

where $\sigma_{i}=\int d \Omega(d \sigma / d \Omega)(1-\cos \theta)^{i}$. Equation $(10)$ is only applicable when $\hbar k_{F} \mu / e \ll 1$. This is true for negative ions in ${ }^{3} \mathrm{He}$ (Table II). The linear term in Eq. (10) is temperature independent and thus seems to explain the missing temperature dependence of the mobility.

Josephson and Lekner ${ }^{25}$ also took into account the frequency dependence of the mobility, which leads to a more complicated form of $F_{0}(K, t)$ than the simple diffusion model. ${ }^{26}$ Numerical solutions of Eq. (6) suggest ${ }^{27}$ that at least the single-relaxation-time approximation of $\mu(\omega)=$ $\mu(0) /\left(1+\omega^{2} \tau^{* 2}\right)$ gives a slightly increasing mobility toward lower temperatures, in contrast to our result. The application of a more detailed scattering function has not yet been performed.

In order to check the nonlinearity in Eq. (10), we measured the velocity of the ion as a function of the drag force up to $15 \mathrm{~cm} / \mathrm{sec}$ at $28.8 \mathrm{bar}$ (cf. Fig. 11). At $3 \mathrm{mK}$ the observed nonlinearity agrees roughly with the prediction. However, in our range of electric fields the effect is too small to make a quantitative test of the theory. In particular, we cannot confirm the predicted temperature dependence of the nonlinear behaviour in the normal liquid.

We carefully checked for possible sources of systematic error in our velocity spectrometer, but found none. The spectrometer was tested with ${ }^{4} \mathrm{He}$; with this liquid we measured considerably shorter traveling times than we have produced in ${ }^{3} \mathrm{He}$. In the ${ }^{4} \mathrm{He}$ experiments we always reproduced the previously measured values of the mobility. ${ }^{5}$

We also measured the ion mobility in the normal phase as a function of pressure, as illustrated in Fig. 5. For each pressure the mobility was obtained by measuring the velocity of ions for different electric fields from 20 to 160 $\mathrm{V} / \mathrm{cm}$ at about $20 \mathrm{mK}$. The velocity was a linear function of the applied electric field. The error bars in Fig. 5 do not contain the $5 \%$ uncertainty in 


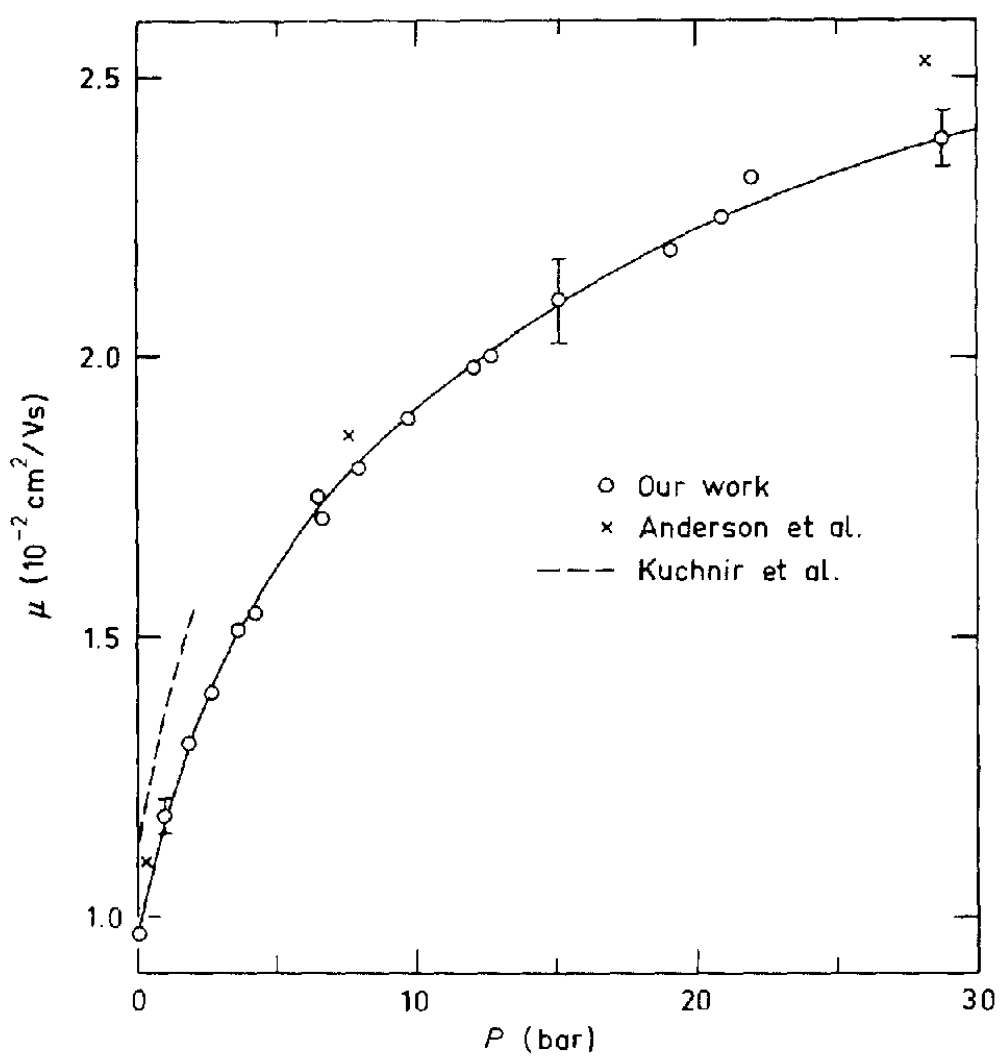

Fig. 5. Normal-phase mobility of the negative ion as a function of pressure: $(0)$, this work; $(\times)$, Ref. 10; (- - ), Ref. 11. The solid line represents a smoothed curve through our data points.

the length of the $5 \mathrm{~mm}$ drift space. If we take this uncertainty into account, the agreement with earlier measurements ${ }^{10,11}$ is rather good.

The principal pressure dependence of the mobility comes from the scattering cross section $\sigma$ [cf. Eqs. (4) and (10)]. If we assume that the differential scattering cross section is isotropic and that $\sigma$ equals the geometrical cross section of the ion bubble, we can use the measured mobility to study the bubble radius $R$ as a function of pressure. In order to eliminate the uncertainty in the length of the drift space, we have plotted in Fig. 6 the radius normalized to the zero-pressure radius. Equation (10) was used in calculating $R$ from the measured mobility.

Following the earlier bubble model theories, ${ }^{2}$ we have made an estimate of the bubble radius. The solid line in Fig. 6 represents a theoretical 


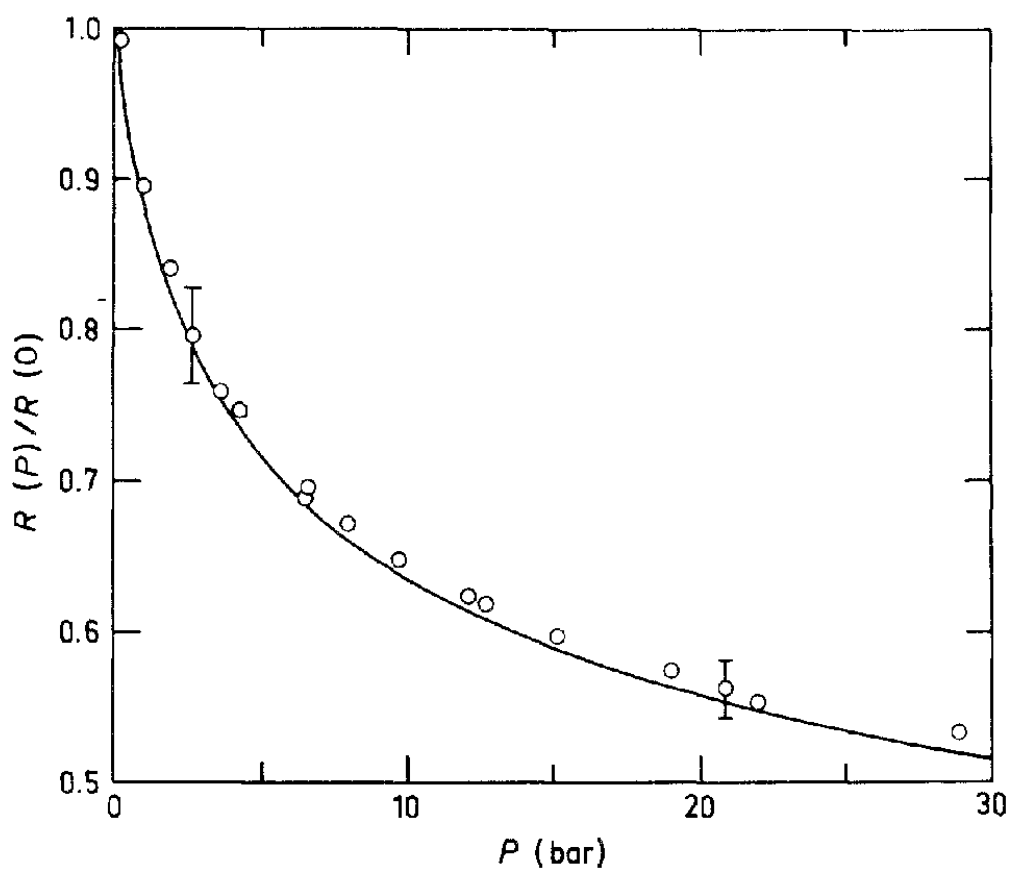

Fig. 6. Normalized bubble radius derived from the mobility data. The solid line represents the theory in the text.

radius which minimizes the total energy of the ion bubble

$$
E(R, P)=E_{e}\left(V_{0}, R\right)+4 \pi \gamma R^{2}+\left(4 \pi R^{3} / 3\right) P
$$

$E_{e}\left(V_{0}, R\right)$ is the electron ground-state energy in a finite square-well potential of radius $R$ and depth $V_{0}, \gamma$ is the surface tension, assumed to be 0.15 $\mathrm{erg} / \mathrm{cm}^{2}$ for all pressures. ${ }^{28}$ The last term represents the volume energy due to the external hydrostatic pressure $P$.

The pressure dependence of $V_{0}$ is taken into account by the formula ${ }^{2}$

$$
V_{0}(P)=\left(2 \pi \hbar^{2} a n / m_{e}\right)\left[1+(9 \pi n / 2)^{1 / 3} a\right]
$$

where $a=0.63 \AA$ is the scattering length of the electron-helium atom interaction potential ${ }^{29}$ and $m_{e}$ is the mass of the electron. Equation (12) describes the repulsive electron-helium interaction by means of a scattering length and includes the two-body correlations of the helium atoms. We have also taken into account the small electrostrictive pressure ${ }^{2}$ which comes from the polarizability of the ${ }^{3} \mathrm{He}$ liquid in the electric field of the electron. $P$ in Eq. (11) is the pressure close to the bubble wall; the actual measured 
pressure $P_{\infty}$ far away from the ion is ${ }^{2}$

$$
P_{\infty}=P-\frac{\alpha n(P) e^{2}}{32 \pi^{2} \varepsilon_{0}[1+n(P) \alpha]^{2} R^{4}}
$$

where $\alpha=2.52 \AA^{3}$ is the polarizability of a ${ }^{3} \mathrm{He}$ atom. ${ }^{2}$

The above model is in good agreement with the normalized radii derived from the measured mobility through Eq. (10). Also, the absolute values $R(0)_{\text {theor }}=21.3 \AA$ and $R(0)_{\text {meas }}=20.3 \pm 2 \AA$ are in reasonable agreement.

\section{MOTION OF NEGATIVE IONS IN SUPERFLUID ${ }^{3} \mathrm{He}$}

\subsection{Results}

In superfluid ${ }^{3} \mathrm{He}$ we found two separate regimes of ion motion: a low-field regime in which the drift velocity $v$ varies linearly with the driving

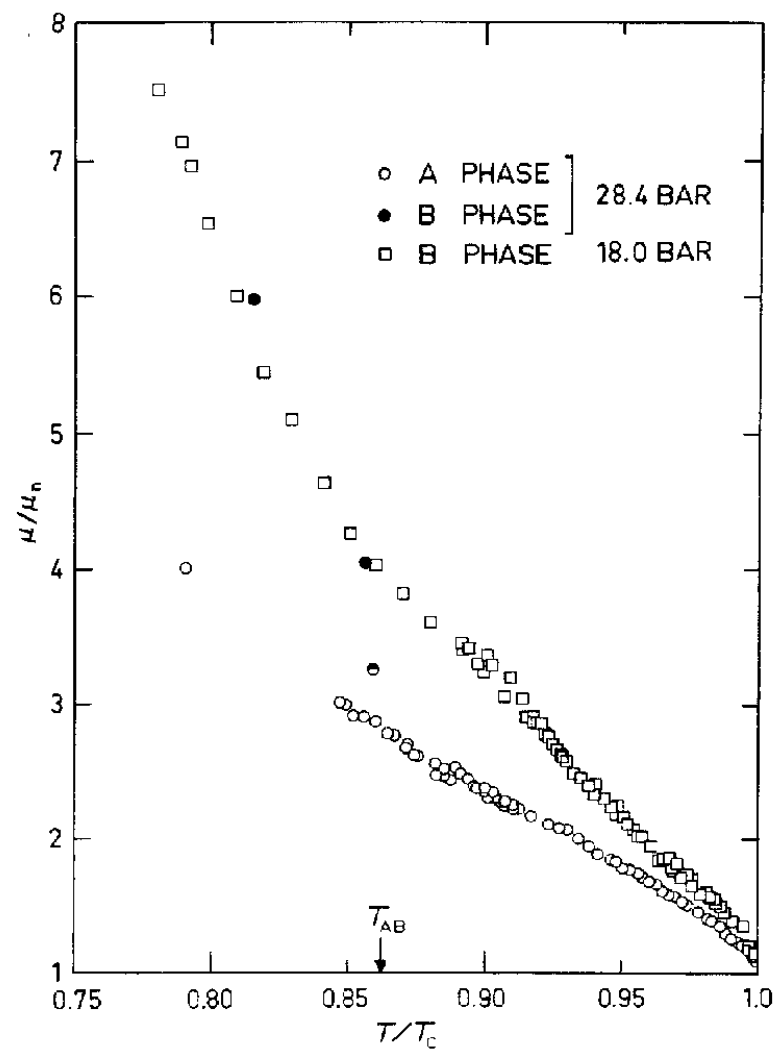

Fig. 7. Ratio of superfluid to normal liquid mobility vs. reduced temperature. 


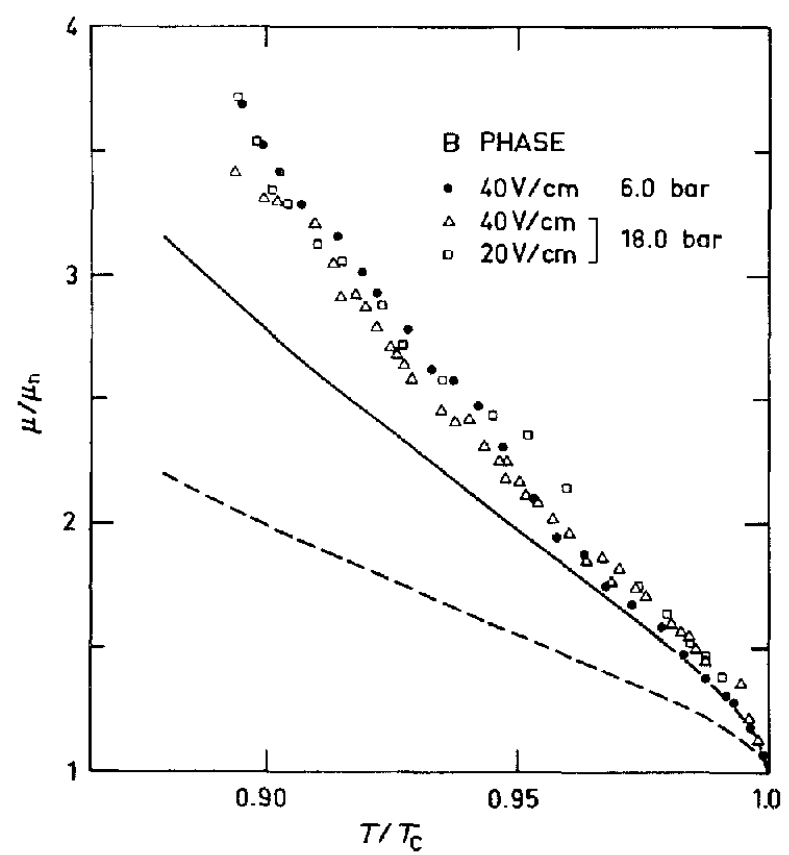

Fig. 8. Normalized mobility in the $\mathrm{B}$ phase as a function of reduced temperature; $(---), \quad \mu / \mu_{n}=\frac{1}{2}\left(e^{\beta \Delta}+1\right),{ }^{31} \quad \Delta=$ $1.07 \Delta_{\mathrm{BCS}} ;(-)$, Ref. $32\left(\Delta=1.07 \Delta_{\mathrm{BCS}}, p_{\mathrm{F}} R=10.3 \hbar\right)$.

field, and a high-velocity regime, in which $v$ is nonlinear. We therefore carried out two types of experiments. We measured the velocity of the ion in a constant electric field $\mathscr{E}$ as a function of temperature. In the linear velocity regime this gave the mobility. Because the smallest $\mathscr{E}$ with which we could detect a signal was only $10 \mathrm{~V} / \mathrm{cm}$ in the normal phase, the requirement of the linearity limited this type of measurement to the range $\mu / \mu_{n} \leqslant 10$ and $T / T_{c}>0.8$. In the second type of experiment we measured the velocity of the ion as a function of the electric field at a constant temperature to study the nonlinear velocity regime. The large heat capacity of our nuclear cooling stage below $T_{c}$ kept the temperature constant within better than $0.01 T_{c}$.

The general behaviour of the mobility in superfluid ${ }^{3} \mathrm{He}$ is shown in Fig. 7. The data were obtaincd at two pressures, 18.0 and 28.4 bar, illustrating the B- and A-phase results, respectively. Summarizing our results, we can state that the mobility in both superfluid phases increases rapidly below $T_{c}$ and that mobility in the $B$ phase is greater than in the A phase. The increase toward lower temperatures reflects the decreasing number of unpaired 


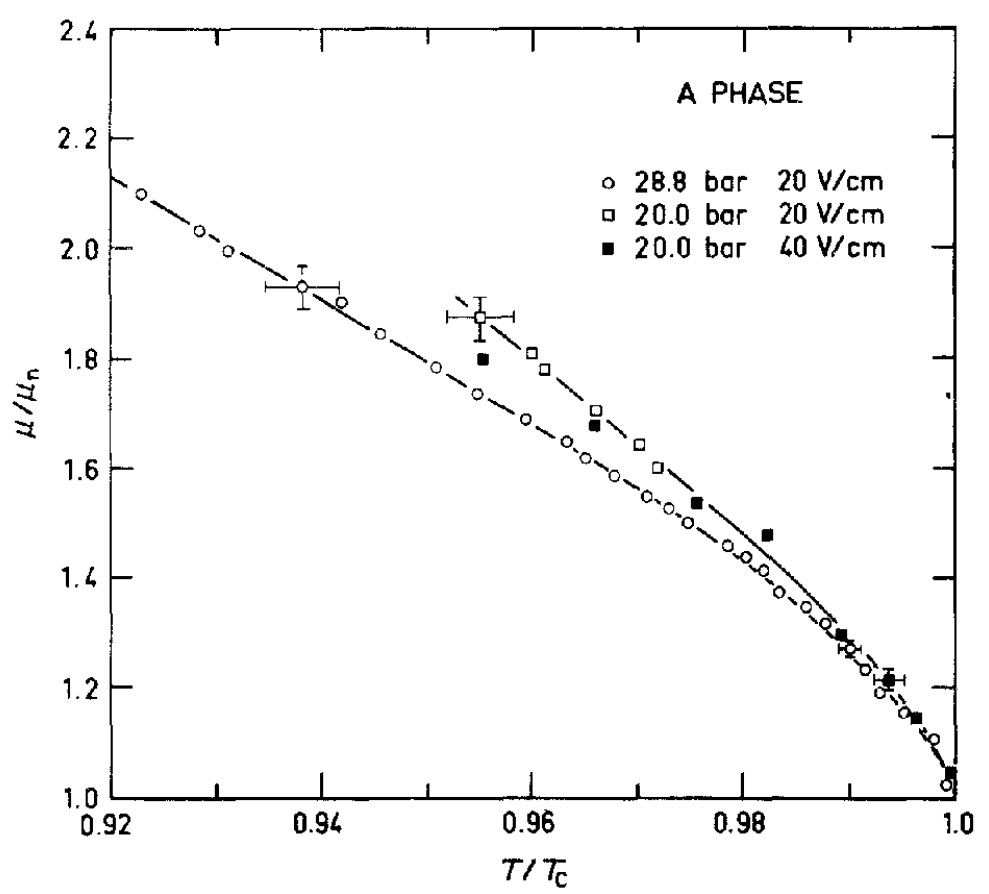

Fig. 9. Normalized mobility in the A phase as a function of reduced temperature. The ions are moving perpendicular to the external magnetic field of $28 \mathrm{mT}$.

quasiparticles and the difference between the A and B phases can qualitatively be related to the different angular dependences of the energy gaps of these phases.

In Figs. 8 and 9 we display the temperature dependence of the mobility at two pressures in both superfluid phases close to $T_{c}$. Unfortunately, the temperature resolution in the $B$ phase was only about $1 \%$. Within this resolution there is no pressure dependence in the B-phase mobility between 6.0 and 18.0 bar close to $T_{c}$. In our A-phase measurements the temperature resolution was about ten times better than in the $B$ phase. The error bars in the A-phase temperature reflect the uncertainty in the calibration of the longitudinal resonance frequency against the platinum thermometer. In the A phase there seems to be a small pressure dependence between 20 and 28.8 bar, with $\mu / \mu_{n}$ increasing toward lower pressures.

In the A phase the high resolution of the thermometer made it possible to study the temperature dependence of the mobility close to $T_{c}$. In Fig. 10 we have plotted $\left(\mu_{n} / \mu-1\right)^{2}$ as a function of the squared longitudinal resonance frequency $\nu_{L}^{2}$ and of the temperature. The experiment was performed at 28.4 bar when the temperature was slowly drifting upward. All 


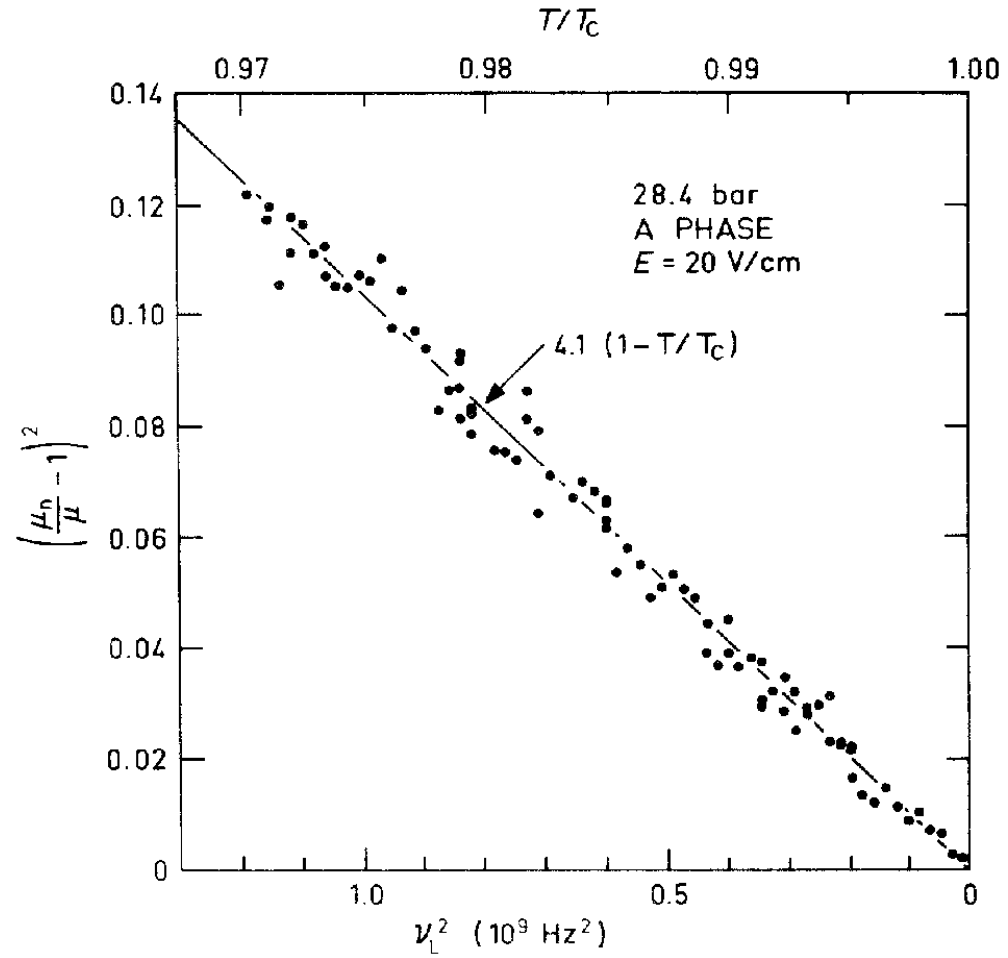

Fig. 10. Temperature dependence of the A-phase mobility close to $T_{c}$. The ions are moving perpendicular to the external magnetic field.

the measured points are shown. We found that $\left(\mu_{n} / \mu-1\right)^{2}$ is a linear function of $1-T / T_{c}$ close to $T_{c}$.

In Table III we have collected the temperature slopes of $\left(\mu_{n} / \mu-1\right)^{2}$ for three different pressures. The large uncertainty in the slopes is partly due to the scatter of the mobility data and partly caused by the calibration of the $\nu_{L}^{\frac{2}{L}}$ against the platinum thermometer. The large error bars make it impossible to resolve a pressure dependence less than $20 \%$; within this uncertainty the slopes are pressure independent.

TABLE III

\begin{tabular}{cccc}
\hline$p$, bar & $\partial\left[\left(\mu_{n} / \mu\right)-1\right]^{2} / \partial \nu_{L}^{2}, \mathrm{~Hz}^{-2}$ & $\partial \nu_{L}^{2} / \partial\left(1-T / T_{c}\right) ; \mathrm{Hz}^{2}$ & $\partial\left[\left(\mu_{n} / \mu\right)-1\right)^{2} / \partial\left(1-T / T_{c}\right)$ \\
\hline 20.0 & $(1.75 \pm 0.2) \times 10^{-10}$ & $(2.80 \pm 0.3) \times 10^{10}$ & $4.9 \pm 1.0$ \\
28.4 & $(1.02 \pm 0.1) \times 10^{-10}$ & $(4.00 \pm 0.2) \times 10^{10}$ & $4.1 \pm 0.6$ \\
28.8 & $(1.10 \pm 0.1) \times 10^{-10}$ & $(4.06 \pm 0.2) \times 10^{10}$ & $4.5 \pm 0.6$ \\
\hline
\end{tabular}




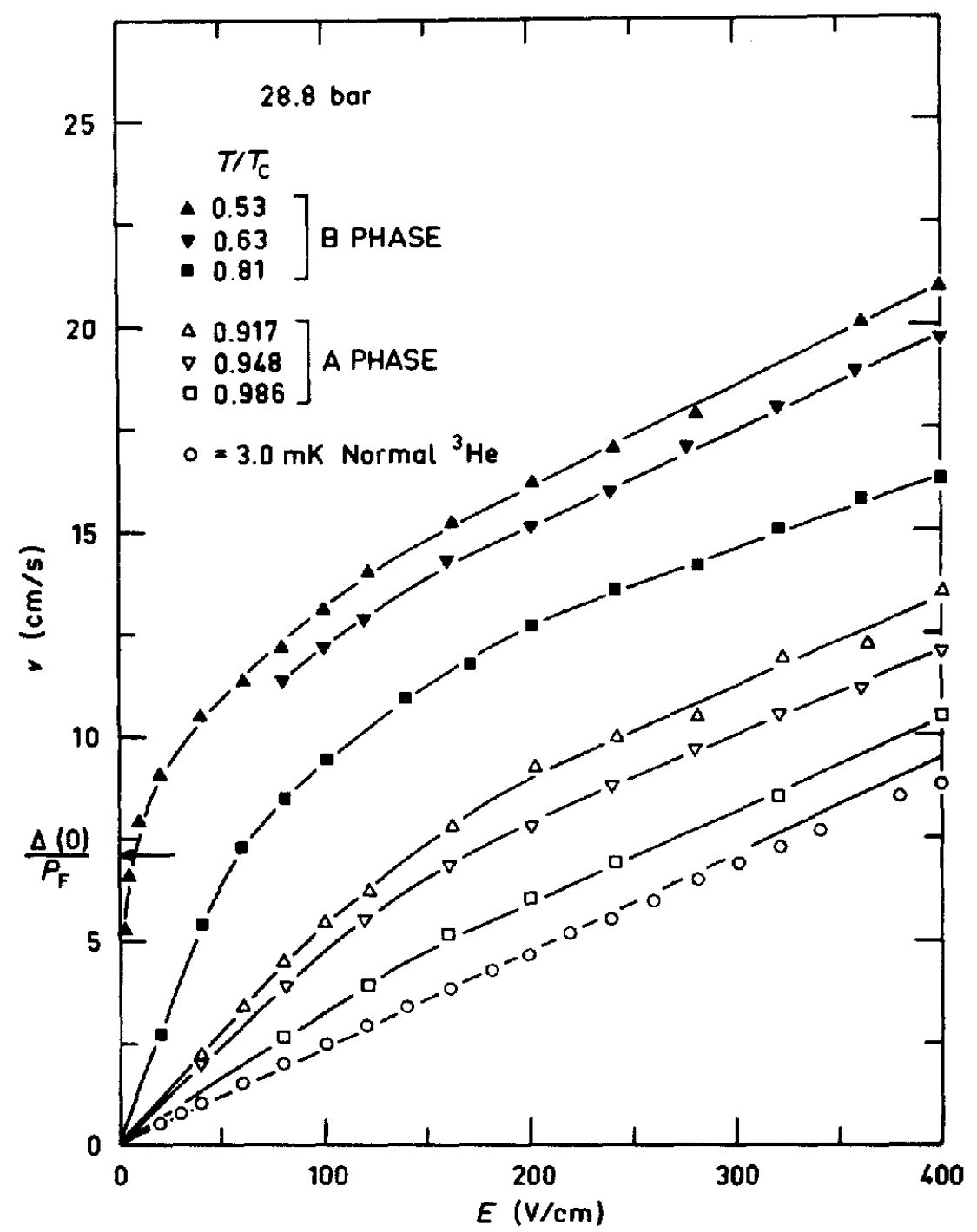

Fig. 11. Velocity of negative ions as a function of the electric field. A tiny nonlinearity is seen already in the normal phase above $200 \mathrm{~V} / \mathrm{cm}$. In the superfluid phases the Landau critical velocity $\Delta / p_{\mathrm{F}}$ is close to the onset of the nonlinear velocity regime.

Our results in the nonlinear velocity regime at 28.8 bar in the A and B phases are presented in Fig. 11. The measurements were extended to electric fields as high as $400 \mathrm{~V} / \mathrm{cm}$. The field dependences of the ion 
velocities in the A and B phases closely resemble each other. The linear regime becomes smaller toward lower temperatures and the velocity curves in the nonlinear regime seem to be roughly parallel to the normal-phase curve.

The onset of the nonlinearity in the ion velocity vs. electric field curve is close to the Landau critical velocity $\Delta / p_{\mathrm{F}}$. This implies that the moving ion breaks quasiparticle pairs, which causes extra resistance for high velocities. The onset is not a sharp change in the slope of the velocity curve. This presumably means that the scattering is inelastic and pair-breaking happens already below the critical velocity $\Delta / p_{\mathrm{F}}$.

\subsection{Discussion}

Contrary to the case of liquid ${ }^{4} \mathrm{He}$ where there is no microscopic picture comparable to the BCS theory, one might believe that in the case of ${ }^{3} \mathrm{He}$, measurements of ion mobility could give some parameters of the theory, such as the energy gap $\Delta$. However, the ion scattering function and the coherence factors of the superfluid phase produce a complicated structure so that the extraction of the internal parameters of the superfluid theory from the measured data is difficult.

The first calculations of the ion mobility in the superfluid phases of ${ }^{3} \mathrm{He}$ did not take advantage of the Josephson-Lekner formalism ${ }^{30}$ or neglected the coherence factors of the superfluid phases. ${ }^{31}$ Both of these were taken into account by Fetter and Kurkijärvi. ${ }^{26}$ They treated the quasiparticle ion scattering in Born approximation and were able to derive the drag force $e \mathscr{E}$ for finite velocities $v$. However, the Born approximation is not valid on the gap edge, where the density of states is infinite and leads to zero mobility.

The correct calculation of the scattering amplitude of a ${ }^{3} \mathrm{He}$ quasiparticle requires that one includes the modification by the superfluidity of intermediate states in the scattering. This was performed by Baym, Pethick, and Salomaa (BPS) ${ }^{32}$ in the zero-field limit and in the elastic scattering approximation $\left[S_{0}(K, \omega)=\delta(\omega)\right]$. They found in the isotropic B phase

$$
\frac{e}{\mu}=-n p_{\mathrm{F}} \int_{-\infty}^{\infty} d \xi\left|\frac{d E}{d \xi}\right| \frac{\partial n}{\partial E} \sigma_{\mathrm{tr}}(\xi)
$$

where $\xi$ is the quasiparticle energy in the normal state, $n(E)=\left(e^{\beta E}+1\right)^{-1}$, $E=\left(\xi^{2}+\Delta^{2}\right)^{1 / 2}, \Delta$ is the energy gap, and $\sigma_{\mathrm{tr}}$ is the momentum transfer cross section. In the normal phase one has $E=\xi$ and readily recovers Eq. (4) for constant scattering cross section.

As pointed out by BPS, the mobility in the superfluid phases is increased by the reduction of the thermally excited quasiparticles as well as 
by a decrease in $\sigma_{\mathrm{tr}}$. The decrease in $\sigma_{\mathrm{tr}}$ is due to the fact that the differential scattering cross section is strongly peaked forward in the superfluid phase and forward scattering processes transfer small momentum. By assuming a constant $\sigma_{\text {tr }}$, they find

$$
\mu / \mu_{n}=\frac{1}{2}\left(e^{\beta \Delta}+1\right)
$$

which was first derived by Bowley. ${ }^{31}$ This result is shown as the dashed line in Fig. 8. It accounts only for about half of the observed increase in the mobility. The solid line in Fig. 8, however, includes the decrease in the momentum transfer cross section, which brings the theory into essential agreement with experiment. The deviation from experiment at lower temperatures presumably indicates the breakdown of the elastic approximation.

A possible pressure dependence of the normalized mobility $\mu_{\mathrm{B}} / \mu_{n}$ can arise from the rather weak dependence ${ }^{32}$ of $\sigma_{\mathrm{tr}} / \sigma_{\mathrm{tr}}^{n}$ on the bubble radius $R$, and from the strong coupling effects on $\Delta(T)$. Because the latter appear to be small, from both theory and experiment, the overall pressure dependence is small and in agreement with measurements.

In the A phase the anisotropy of the energy gap makes the calculation of mobility more complicated. The full calculation is not yet available. Therefore the behavior of $\mu_{\mathrm{A}}$ in the Ginzburg-Landau region is presently analyzed by assuming a constant $\sigma_{\mathrm{tr}}{ }^{33}$ To first order in $\Delta_{\max }$, the maximum value of the gap, the parallel component $\mu_{\|}(\mathbf{v} \| \boldsymbol{l})$ and the perpendicular component $\mu_{\perp}(\mathbf{v} \perp \boldsymbol{l})$ are

$$
\begin{gathered}
\mu_{n} / \mu_{\|} \simeq 1-0.42 \beta \Delta_{\max } \\
\mu_{n} / \mu_{\perp} \simeq 1-0.47 \beta \Delta_{\max }
\end{gathered}
$$

In weak coupling theory one has $\Delta_{\max }^{2}=\frac{5}{4} \Delta_{\mathrm{BCs}}^{2}$. Because of strong coupling effects the energy gap is enhanced by a factor $\left[\left(\Delta C / C_{N}\right) /\left(\Delta C / C_{N}\right)_{\mathrm{BCs}}\right]^{1 / 2}$, where $\Delta C / C_{N}$ is reduced specific heat discontinuity at $T_{c}{ }^{34}$ The measured $\Delta C / C_{N}$ at melting plane is $2.0,{ }^{35}$ which gives for averaged mobility

$$
\left\{\left[\left(\mu_{\perp}+\mu_{\|}\right) / 2 \mu_{n}\right]-1\right\}^{2}=3.3\left(1-T / T_{c}\right)
$$

At 20 bar the enhancement factor is about $25 \%$ smaller than at the melting pressure, ${ }^{21}$ and gives 2.5 instead of 3.3. These coefficients should be compared with the measured values $4.9 \pm 1.0$ at 20 bar and $4.1 \pm 0.6$ at 28.8 bar (Table III). The measured values are higher than the theoretical ones. Part of the difference may be due to a possible reduction of $\sigma_{\text {tr }}$ in the A phase.

The nonlinear velocity regime in the B phase has been studied by Fetter and Kurkijärvi ${ }^{26}$ using the Born approximation and the elastic scattering limit. In Fig. 12 we compare our results with the calculated velocity at $T / T_{c}=0.45$ and 0.81 . Even though the Born approximation leads to 


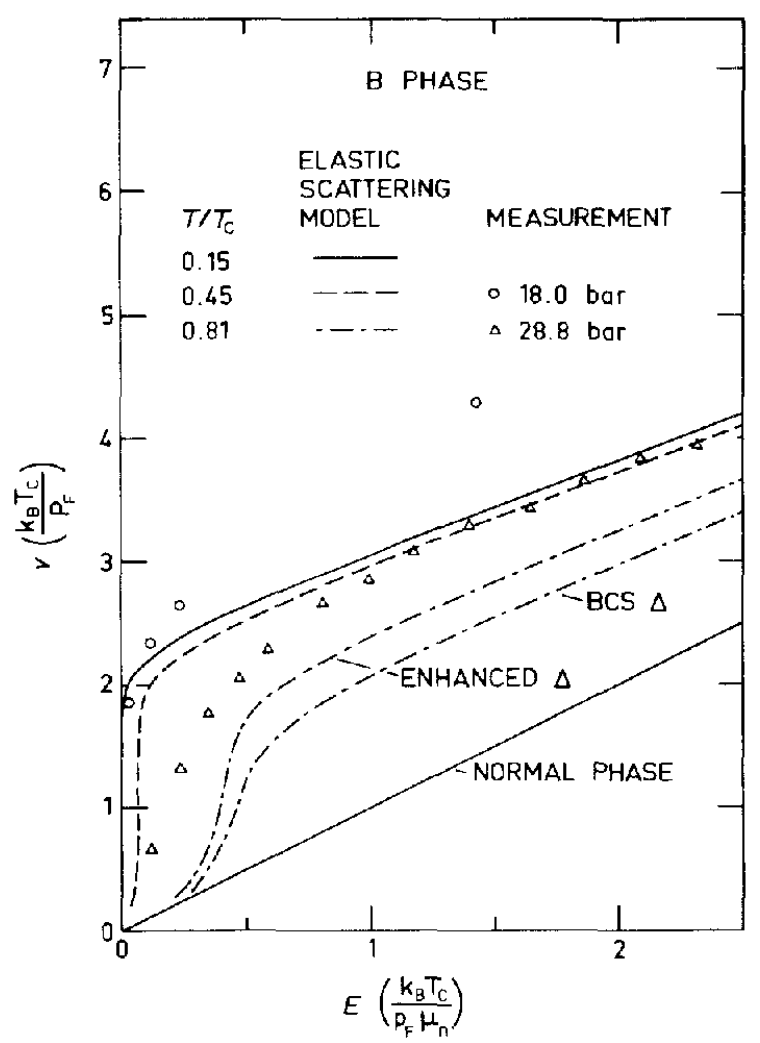

Fig. 12. Velocity vs. the electric field in the $B$ phase compared with the elastic scattering model. ${ }^{26}$ The calculations are based on the BCS energy gap except at $T / T_{c}=0.81$ and at $28.8 \mathrm{bar}$, wherc $\Delta_{\text {enhanced }}=1.19 \Delta_{\mathrm{BCS}}$ is also shown for comparison. The Born approximation and elastic scattering model lead to a scaling of electric field $E$ and velocity $v$ for different pressures.

diverging scattering integrals at the zero-velocity limit, the calculated curves resemble qualitatively the measured data for high velocities: The bending over happens at $v \approx v_{c}=\Delta / p_{\mathrm{F}}$ and for $v>v_{c}$ the drag force approaches the normal-state value. In the calculated curves the high-velocity behavior is mainly determined by the pair-breaking effect of the moving ion.

\section{CONCLUSION}

We have carried out a brief survey of the negative ion motion in normal and superfluid ${ }^{3} \mathrm{He}$. In the normal phase we find strictly temperatureindependent mobilities at all pressures. The present theories cannot fully 
explain this behavior. We also studied nonlinear effects in $v$ without conclusive results. Further investigations in this direction would increase our understanding of inelastic scattering of the quasiparticles by an ion.

In the superfluid phases we observed a steep increase of the mobility below $T_{c}$ and our data seem to agree with the present theory. ${ }^{32,33}$ The high-velocity regime can be qualitatively described as a pair-breaking effect of the ion but quantitative calculations ${ }^{26}$ are not in satisfactory agreement with our results.

It would be interesting to extend the measurements to much smaller electric fields in order to obtain a field-independent mobility also for $T / T_{c}<0.8$. Space charge effects, however, will cause a serious problem. In addition, the possibility of an anisotropic mobility in the A phase remains to be investigated

For a deeper understanding of the motion of a heavy impurity in superfluid ${ }^{3} \mathrm{He}$ it is necessary to make measurements with positive ions as well. These have a smaller effective mass and radius than negative ions and thus the mobility measurement could give additional information about inelastic scattering of quasiparticles by the impurity.

\section{APPENDIX. VELOCITY MEASUREMENTS IN THE PRESENCE OF SPACE CHARGE EFFECTS}

A one-dimensional model is a good approximation in our electrode geometry. We assume that the ion cloud is a charge layer moving in an external field $\mathscr{E}_{\text {ext }}=V / l$ in the drift space of length $l$ between $\mathrm{G} 1$ and $\mathrm{G} 2$ (cf. Fig. 13). Let the thickness of the layer be $d$ and let $\rho$ be the uniform charge density. The boundary conditions for the one-dimensional potential problem are: $V_{1}=0$ and $V_{2}=V$. If $\mathscr{E}_{1}$ and $\mathscr{E}_{2}$ are the electric fields on both sides of the layer, we obtain the following equations (MKSA units):

$$
\begin{gathered}
\mathscr{E}_{1}-\mathscr{E}_{2}=\rho d / \varepsilon_{0}=\mathscr{E}_{\text {int }} \\
\mathscr{E}_{2}(z-d / 2)+\left(\mathscr{E}_{1}+\mathscr{E}_{2}\right) d / 2+\mathscr{E}_{1}(l-z-d / 2)=V
\end{gathered}
$$

After solving for $\mathscr{E}_{1}$ and $\mathscr{E}_{2}$, we calculate the traveling times $t_{f}$ and $t_{t}$ of the front and trailing edges, respectively, by integrating

$$
t=\frac{1}{\mu} \int_{0}^{l} \frac{d z}{\mathscr{E}(z)}
$$

and by assuming that the front edge is driven by $\mathscr{E}_{1}$ and the trailing edge by 


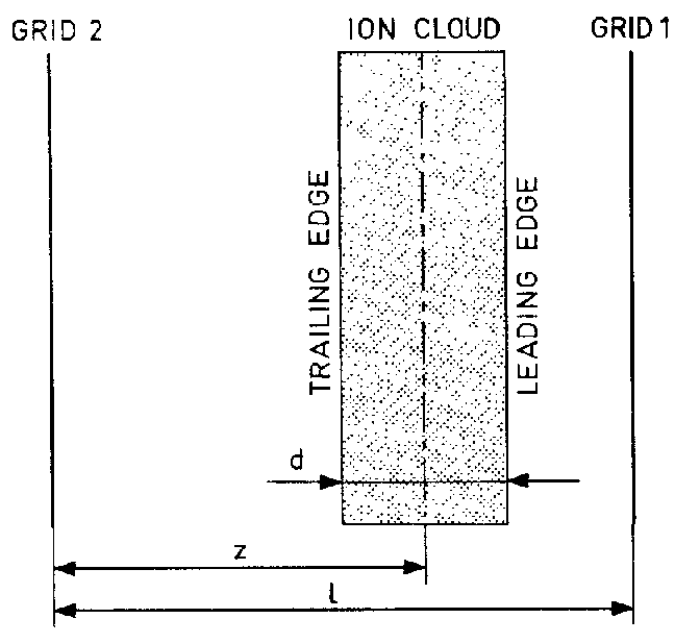

Fig. 13. Schematic illustration of the moving charge layer between grids 1 and 2 .

$\mathscr{E}_{2}$. We obtain

$$
\begin{aligned}
& t_{f}=\frac{l^{2}}{\mu V} \frac{\ln (1+x)}{x} \\
& t_{t}=\frac{l^{2}}{\mu V} \frac{|\ln (1-x)|}{x}
\end{aligned}
$$

where $x=\mathscr{E}_{\text {int }} / \mathscr{E}_{\text {ext }}=\left(\rho d / \varepsilon_{0}\right) /(V / l)$.

By measuring $t_{f}$ and $t_{t}$ the unknown $x$ can be eliminated and the mobility $\mu$ can be calculated. We found that our data were in agreement with this model. In all our measurements $x$ was smaller than 0.2

\section{ACKNOWLEDGMENTS}

The authors are indebted to Taisto Haavasoja and Leila Rehn for their help during these measurements.

We have had valuable discussion and correspondence with Roger Bowley, Sandy Fetter, Olli Lounasmaa, Chris Pethick, Dierk Rainer, Martti Salomaa, Joe Serene, Toshio Soda, Matti Vuorio, and especially with Juhani Kurkijärvi.

\section{REFERENCES}

1. A. I. Ahonen, J. Kokko, O. V. Lounasmaa, M. A. Paalanen, R. C. Richardson, W. Schoepe, and Y. Takano, Phys. Rev. Lett. 37, 511 (1976). 
2. A. L. Fetter, in The Physics of Liquid and Solid Helium, K. H. Bennemann and J. B. Ketterson, eds. (Wiley, New York, 1976), pp. 242-305, and references therein.

3. J. Poitrenaud, in Liquid and Solid Helium, C. G. Kuper, S. G. Lipson, and M. Revzen, eds. (Wiley, New York, 1974), pp. 255-258.

4. W. Schoepe and G. W. Rayfield, Phys. Rev. A 7, 2111 (1973); J. R. Broomall, W. D. Johnson, and D. G. Onn, Phys. Rev. B 14, 2819 (1976).

5. L. Meyer and F. Reif, Phys. Lett. 5, 1 (1960).

6. K. W. Schwarz, Phys. Rev. A 6, 837 (1972).

7. G. W. Rayfield and F. Reif, Phys. Rev. Lett. 11, 305 (1963).

8. G. W. Rayfield, Phys. Rev. Lett. 16, 934 (1966).

9. D. R. Allum, P. V. E. McClintock, A. Phillips, and R. M. Bowley, to be published.

10. A. C. Anderson, M. Kuchnir, and J. C. Wheatley, Phys. Rev. Lett. 168, 261 (1968).

11. M. Kuchnir, P. R. Roach, and J. B. Ketterson, Phys. Rev. A 2, 262 (1970).

12. P. V. E. McClintock, J. Low Temp. Phys. 11, 277 (1973).

13. A. I. Ahonen, P. Berglund, M. Haikala, M. Krusius, O. V. Lounasmaa, and M. A. Paalanen, Cryogenics 16, 521 (1976).

14. F. E. Moss, in Methods of Experimental Physics, R. V. Coleman, ed. (Academic Press, New York and London, 1974), Vol. 11, pp. 443-484.

15. B. L. Henson, Phys. Rev. 135, A1002 (1964).

16. B. Halpern and R. Gomer, J. Chem. Phys. 51, 1048 (1969).

17. C. A. Mead, J. Appl. Phys. 32, 646 (1961).

18. D. G. Onn, P. Smejtek, and M. Silver, J. Appl. Phys. 45, 119 (1974).

19. S. Brunauer, P. H. Emmett, and E. Teller, J. Am. Chem. Soc. 60, 309 (1938).

20. M. I. Aalto, H. K. Collan, R. G. Gylling, and K. O. Nores, Phys. Lett. 41A, 469 (1972).

21. A. I. Ahonen, M. Krusius, and M. A. Paalanen, J. Low Temp. Phys. 25, 421 (1976).

22. D. D. Osheroff, W. J. Gully, R. C. Richarson, and D. M. Lee, Phys. Rev. Lett. 29, 920 (1972).

23. A. I. Ahonen, M. Krusius, and M. A. Paalanen, in Low Temperature Physics-LT14, M. Krusius and M. Vuorio, eds. (North-Holland, Amsterdam, 1975), Vol. 1, p. 107.

24. R. Abe andK. Aizu, Physics 123, 10 (1961); R. C. Clark, Proc. Phys. Soc. (London) 82, 785 (1963); G. T. Schappert, Phys. Rev. 168, 162 (1968).

25. B. D. Josephson and J. Lekner, Phys. Rev. Lett. 23, 111 (1969).

26. A. L. Fetter and J. Kurkijärvi, Phys. Rev, B, to be published.

27. R. M. Bowley, J. Phys. C 4, L207 (1971).

28. D. R. Lovejoy, Can.J. Phys. 33, 49 (1955); K. N. Zinoveva, Sov. Phys._JETP 2, 774 (1956).

29. L. L. Tankersley, J. Low Temp. Phys. 11, 451 (1973).

30. T. Soda, Prog. Theor. Phys. 53, 903 (1975).

31. R. M. Bowley, J. Phys. C 2, L151 (1976).

32. G. Baym, C. J. Pethick, and M. Salomaa, Phys. Rev. Lett. 38, 845 (1977).

33. C. J. Pethick, private communication.

34. A. J. Leggett, Rev. Mod. Phys. 47, 331 (1975).

35. W. P. Halperin, C. N. Archie, F. B. Rasmussen, T. A. Alvesalo, and R. C. Richardson, Phys. Rev. B 13, 2124 (1976). 